and urinary benzene (U-Benz) to detect low level occupational and environmental exposure to benzene.

Methods We monitored airborne benzene by personal air sampling, and U-Benz, s-PMA, $t, t-\mathrm{MA}$ and cotinine (U-Cotinine) in spot urine samples, collected at 8 am and $8 \mathrm{pm}$, in 32 oil refinery workers and 65 subjects, randomly selected among the general population of urban and suburban Cagliari, Italy.

Results The median concentration of airborne benzene was $25.2 \mu \mathrm{g} / \mathrm{m}^{3}$ in oil refinery workers, and $8.5 \mu \mathrm{g} / \mathrm{m}^{3}$ in the general population subgroup. U-Benz in morning and evening samples was significantly more elevated among oil refinery workers than the general population subgroup $(p=0.012$, and $p=7.4$ $\mathrm{x} 10^{-7}$, respectively) and among current smokers compared to non-smokers ( $p=5.2 \times 10^{-8}$, and $p=5.2 \times 10^{-5}$ respectively). Benzene biomarkers and their readings in the two sampling phases were well correlated to each other. The Spearman's correlation coefficient with airborne benzene was significant for $\mathrm{U}$-Benz in the evening sample, but not for $t, t$-MA and $s$-PMA in either sampling. Morning U-Cotinine excretion showed a good correlation with U-Benz in the morning and in the evening sampling $(p<0.001)$, and with $s$-PMA in the evening sample $(p<0.001)$, but not with $t, t$-MA in either samplings. $t, t$-MA in the evening sample was the only biomarker showing a moderate inverse correlation with BMI $(\mathrm{p}<0.05)$. The multiple regression analysis adjusting by BMI and number of cigarettes smoked during the day confirmed the results of the univariate analysis.

Discussion Our results suggest that unmetabolised U-Benz would allow a more reliable biomonitoring of low-level exposure to benzene than $s$-PMA and $t, t$-MA.

\section{DEVELOPMENT OF A PESTICIDE CROP/LIVESTOCK EXPOSURE MATRIX USING DATA FROM THREE AGRICULTURAL COHORT STUDIES IN THE AGRICOH CONSORTIUM}

\begin{abstract}
${ }^{1} \mathrm{~L}$ S Schinasi, ${ }^{2}$ Beane Freeman, ${ }^{3}$ Baldi, ${ }^{4}$ Kromhout, ${ }^{5}$ Lebailly, ${ }^{6}$ Nordby, ${ }^{7}$ Kjaerheim, ${ }^{4}$ Brouwer, ${ }^{2}$ Alavanja, ${ }^{1}$ Schuz, ${ }^{1}$ Leon. 'International Agency for Research on Cancer, Lyon, France; ${ }^{2}$ National Cancer Institute, Rockville, United States of America; ${ }^{3}$ Universite Victor Segalen, Bordeaux 2, Bordeaux, France; ${ }^{4}$ Institute for Risk Assessment Sciences, Utrecht University, Utrecht, The Netherlands; ${ }^{5}$ Universite de Caen, Caen Cedex 05, France; ${ }^{6}$ National Institute of Occupational Health, Oslo, Norway; ${ }^{7}$ Cancer Registry of Norway, Institute of Population Based Cancer Research, Oslo, Norway
\end{abstract}

\subsection{6/oemed-2013-101717.130}

Objectives Excess risk of lympho-hematopoietic malignancies has been documented in farmers. Although numerous studies have investigated associations of various pesticides with lymphohematopoietic malignancies, results have been inconsistent. A crop/livestock-exposure matrix for estimating exposures to pesticides is being developed for a pooled analysis of lymphohematopoietic malignancies in farmers.

Methods Data arise from the United States Agricultural Health Study (AHS), the French Agriculture and Cancer Study (AGRICAN), and the Cancer in the Norwegian Agricultural Population Study. When study data are unavailable, historical information on region and crop/livestock specific pesticide use are being gathered from agricultural records, experts, and the French PESTIMAT matrix. Selection of matrix axes is based on information availability and importance in predicting pesticide exposures; of crop/ livestock categories is based on major commodities in each country and common production across or frequent production within the cohorts; and of chemical groups is based on frequency of use and a priori expectations of associations with lympho-hematopoietic malignancies.

Results The cohorts contain varying levels of exposure information. AHS contains information on ever lifetime chemical use for 52,394 private applicators. AGRICAN contains data on crops/ livestock ever produced in the lifetime of 182,132 farmers. For each 10-year agricultural census from 1969-1989, the Norwegian cohort contains data on crops/livestock farmed by 248,000 farmers and spouses. Matrix axes will be defined by time period, crop/ livestock produced and pesticide chemical group. Matrix cells will be filled with pesticide use information by chemical group and, if possible, chemical substance. Crop/ livestock groups will include major categories such as corn, grains, potatoes, soybeans, tobacco, vineyards, poultry, beef cattle, dairy cattle, swine and sheep/goats.

Conclusions This work demonstrates the development of a pesticide exposure matrix using pooled data from multiple countries, which will be used for the largest evaluation of pesticides and lympho-hematopoietic malignancies to date.

\section{RESPIRABLE QUARTZ EXPOSURE ON TWO MEDIUM- SIZED FARMS IN SOUTHERN MOZAMBIQUE}

'A J Swanepoel, ${ }^{2}$ Rees, ${ }^{3}$ Mirembo. 'University of the Witwatersrand, Johannesburg, South Africa; ${ }^{2}$ National Institute for Occupational Health, Johannesburg, South Africa; ${ }^{3}$ University of Eduardo Mondlane, Maputo, Mozambique

\subsection{6/oemed-2013-101717.131}

Background Little is known about the potential for overexposure to respirable quartz in farming, in most parts of the world.

Objectives To measure respirable dust and quartz exposure of tractor operators on two medium-sized dry climate farms.

Methods This is a descriptive cross-sectional study design of dust exposure of four tractor operators. Farms were selected by convenience sampling. The MDHS 14/3 and FTIR MDHS 101 HSE methods were used to measure dust and to analyse the mass of quartz in dust, respectively.

Results Seventy respirable dust measurements were done. Respirable dust and quartz ranged from 0.01 to $2.88 \mathrm{mg} / \mathrm{m}^{3}$ and 0.001 to $0.30 \mathrm{mg} / \mathrm{m}^{3}$. All operators had at least one respirable quartz exposure above $0.1 \mathrm{mg} / \mathrm{m}^{3}$. Only $17 \%$ of respirable quartz concentrations were lower than the ACGIH TLV of $0.025 \mathrm{mg} / \mathrm{m}^{3}$.

Conclusion The potential for over-exposure to respirable quartz was demonstrated. There was a great deal of variability in these farms which has implications for sampling strategies for dust in farming.

\section{Session: R. Musculoskeletal disease}

\section{REPEAT WORKER'S COMPENSATION CLAIMS: A PROSPECTIVE ANALYSIS IN ONTARIO, CANADA AND VICTORIA, AUSTRALIA}

${ }^{1}$ A Mustard, ${ }^{1}$ Etches, ${ }^{2}$ Ruseckaite, ${ }^{2}$ Collie. ${ }^{1}$ Institute for Work \& Health, Toronto, Canada; ${ }^{2}$ Monash University, Victoria, Australia

10.1136/oemed-2013-101717.132

Objective Many of the demographic and labour market characteristics of workers that are associated with the risk of work- 
related injury and disability are well-known. However, the characteristics of workers who have multiple workers' compensation claims in a defined period of time is less clearly understood. This study estimates the extent to which industries, occupations or injury characteristics are associated with a higher risk for the registration of repeat claims. We present a parallel analysis of Ontario, Canada, and Victoria, Australia to determine if there aredifferences in repeat claim patterns between these two jurisdictions.

Methods First lost-time claims of greater than ten days of wagereplacement benefits were identified in the period January 2000 to December 2004 using administrative records of workers' compensation claims. Claimants were followed prospectively for subsequent claims for 5 years from the date of the first claim to estimate the risk for a repeat claim by industry, occupation and injury characteristics.

Results The five year cumulative incidence of an initial claim in Victoria (14.5/1000 person years) was twice the rate observed in Ontario (7.6/1000 person years) The probability of a second lost-time claim of ten days or longer duration within 5 years of the date of the first injury was 0.25 in Victoria and 0.12 in Ontario. The probability of a second claim was related to age, sex, nature of injury, occupation and industry. The distribution of characteristics of second injuries was similar between Ontario and Victoria.

Conclusion This study has used population-based work disability insurance records to compare the incidence of repeat compensation claims in two jurisdictions. Despite differences in scheme benefit policies, this study has found broadly similar patterns in the two settings. The potential for causal inference regarding risk factors for repeat claims is discussed.

\section{RESULTS OF A MYOTONOMETRICAL MUSCLE STUDY IN PATIENTS WITH WORK-RELATED HAND OVERLOAD DISORDER}

${ }^{1} V$ P Pille, ${ }^{2}$ Oha, ${ }^{1}$ Tint. ' Tallinn University of Technology, Tallinn, Estonia; ${ }^{2}$ Tartu University, Tartu, Estonia

\subsection{6/oemed-2013-101717.133}

Introduction Implementation of the new myotonometrical diagnostic method in occupational medicine enables non-invasive, easy and quick evaluation of the functional state of muscles.

Aims Use of the myotonometrical method to study hand muscles in patients with work-related hand overload disorders, and analysing the correlations of the results of myotonometrical testing broken down by various diagnosed disease groups. To analyse whether myotonometry could be used in occupational medicine as a method for evaluating the functional state of muscles.

Method 25 patients with diagnosed work related carpal tunnel syndrome or/and tenosynovitis in the right wrist were studied. Diseases were diagnosed using ENMG and ultrasound examinations.

The mechanical characteristics of frequency, stiffness and elasticity of right hand muscle $m$. abductor pollicis brevis were recorded by means of the Myoton-3 hand-held myotonometer.

For data processing, the Stata program and the Student T-test were used.

Results An average age of patients 54, standard deviation 6.6, $\min 42$ and max 67 , the length of service 24.8 years, the average workday length 6.8 hours. Significant changes regarding muscle decrement and stiffness were apparent in affected workers as compared to the norm.

In subjects with the diagnosis of abd poll br decrement vs norm, the average values were 2.0 and $1.6, \mathrm{p}<0.001$; i.e. there was a significant difference. Stiffness differed significantly from the norm: 311.4 vs $348.8, \mathrm{p}=0.004$.

Conclusion In case of carpal tunnel syndrome and tenosynovitis due to overload of the right wrist area, also signs of an overload of the abductor pollcis muscle can be detected by myotonometric testing. Consequently, myotonometrical muscle study could be used as an auxiliary diagnostic method as well as an initial study in the course of physical examination in order to evaluate possible hand muscle overload.

\section{VARIABILITY OF WORK-RELATED MUSCULOSKELETAL SYMPTOMS, WORK EXPOSURES, AND CONDITIONS BY EMPLOYMENT SETTING AMONG UNITED STATES CERTIFIED ATHLETIC TRAINERS}

${ }^{1}$ L Kucera, 'Lipscomb, 'Dement, ${ }^{2}$ Hootman, ${ }^{3}$ Roos. ${ }^{1}$ Duke University Medical Center, Durham, United States of America; ${ }^{2}$ Centers for Disease Control and Prevention, Atlanta, GA, United States of America; ${ }^{3}$ University of North Carolina, Chapel Hill, NC, United States of America

\subsection{6/oemed-2013-101717.134}

Objectives In addition to colleges and schools, certified athletic trainers (ATs) are now found in hospitals, clinics and other settings including industry. We describe ATs musculoskeletal symptoms, and work exposures and conditions in different settings.

Methods We administered online surveys to a random sample of 10,000 US ATs. Participants $(n=1826)$ estimated the frequency they performed selected work tasks and rated task-related stress [scale 0 (no problem) to 10 (major problem)]. Musculoskeletal symptoms (MS) that interfered or prevented work in the last year was reported by body region as well as frequency with which ten protective strategies were used to reduce work-related physical strain [scale 1 (never) to 5 (always)].

Results ATs commonly worked in schools (67\%) and clinics or hospitals (20\%). Prevalence of MS that interfered/prevented work was $27.2 \%$ (95\% CI: 25.1\%, 29.3\%); prevalence was highest in the low back (13.7\%) and wrist/hand (7.0\%). Back pain was higher in schools (14.7\%) and other (12.9\%) settings compared to clinics/hospitals (9.7\%). ATs in schools more frequently performed taping/wrapping, used treatment modalities, handled/moved equipment, cared for multiple patients at once, worked under time constraints, awkward positions or sustained positions, and inclement weather conditions. School ATs rated physical stress of handling/moving equipment (mean $=4.9$ ), working in same position (4.8), inclement weather conditions (4.0), being short staffed (5.0) and work scheduling issues (5.4) higher than clinic/hospital ATs. Mental stress due to understaffing (mean $=4.9$ ), increased workload (5.0), irregular schedules (5.1), and long work weeks (5.8) were also rated higher by school ATs. School ATs reported always or almost always getting help with patient transfers, asking others to perform physically stressful tasks, and using electrotherapy instead of manual techniques to avoid injury aggravation.

Conclusions Work tasks, physical and mental stressors, and use of protective strategies varied by work setting. Preventive measures need to consider variability across work settings. 